\title{
Pd-Catalyzed Direct Modification of an Anti-Alzheimer's Disease Drug: Synthesis and Biological Evaluation of $\alpha$-Aryl Donepezil Analogues
}

Lin-Xi Wan, Shi-Xing Miao, Zhen-Xiang He, Xiaohuan Li, Xian-Li Zhou,* and Feng Gao*

Cite This: ACS Omega 2021, 6, 23347-23354

Read Online

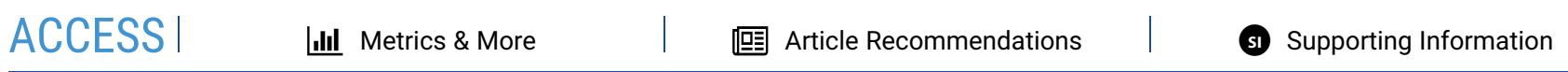

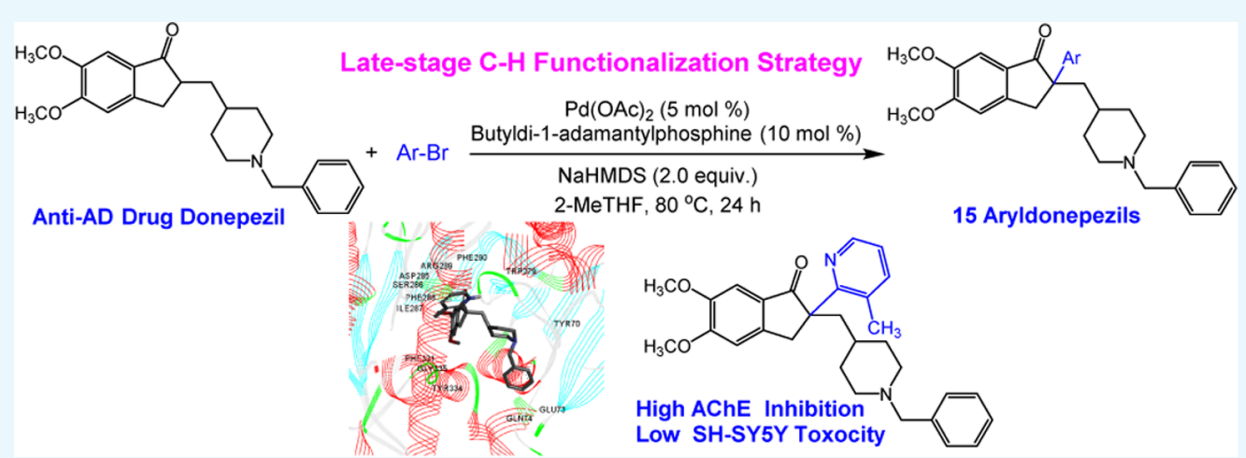

ABSTRACT: Palladium/BuAd 2 P efficiently catalyzed the direct $\alpha$-arylation of ketone in the anti-Alzheimer's disease drug donepezil, leading to 15 aryldonepezil analogues exhibiting high selective inhibition of acetylcholinesterase (AChE). The cell-based assays revealed that the 3-methylpridinyl analogue (12) shows significantly lower toxicity compared to donepezil and remarkable neuroprotective activity against $\mathrm{H}_{2} \mathrm{O}_{2}$-induced damage in SH-SY5Y cells. Docking results of compound $\mathbf{1 2}$ also interpreted the possible mechanism of the selective inhibition between $\mathrm{AChE}$ and butyrylcholinesterase (BuChE).

\section{INTRODUCTION}

Alzheimer's disease $(\mathrm{AD})$ is a severe age-dependent neurodegenerative disorder, with no cure so far. ${ }^{1-4} \mathrm{AD}$ seriously endangers the physical and mental health, causing deep suffering to patients. It has attracted widespread attention from medical circles in various countries. Thus, finding better strategies for the treatment of $\mathrm{AD}$ has become a worldwide endeavor. ${ }^{5} \mathrm{Up}$ to now, only five drugs have been approved by FDA for the treatment of $\mathrm{AD}$, including tacrine, rivastigmine, galantamine, donepezil, and menantine (Figure 1).

Donepezil is a second-generation specific reversible central acetylcholinesterase (AChE) inhibitor ${ }^{6,7}$ for $\mathrm{AD}$ therapy with many side effects, such as nausea, vomiting, diarrhea, fatigue, muscle cramps, lack of appetite, and so forth. Thus, the development of donepezil derivatives with high AChE inhibitory activity and low cell toxicity has drawn immense attention. Several donepezil derivatives have been prepared to find better anti-AD compounds. Previous modifications of donepezil only focused on the $N$-benzylpiperidine group ${ }^{8-11}$ or the terminal benzene ring. ${ }^{12}$ Besides these, donepezil was also conjugated with different moieties like tacrine ${ }^{13}$ and huperzine A. ${ }^{14,15}$ All the previous modifications used the de novo synthesis pathway, in which various fragments were introduced in the beginning, and so, more synthetic steps were necessary for the preparation of structural diverse donepezil derivatives.

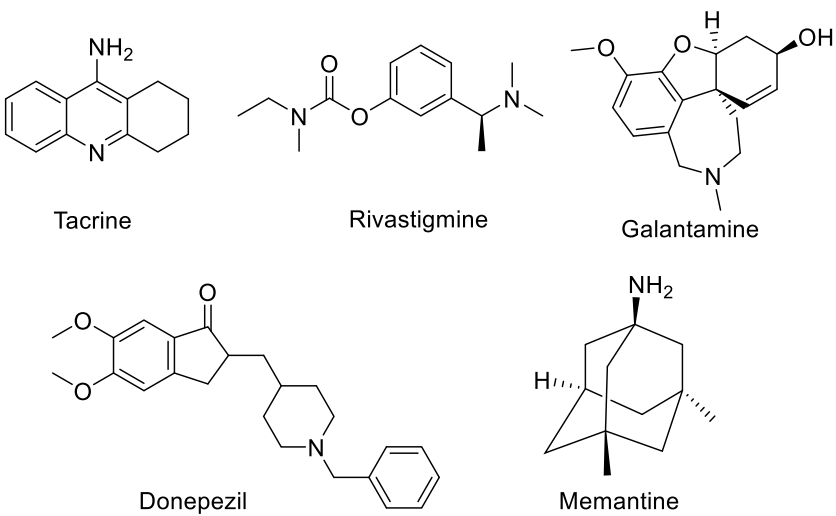

Figure 1. Approved drugs for the treatment of $\mathrm{AD}$.

Aromatic groups like phenyl, pyridinyl, quinolinyl, and so forth are important pharmacophores in the field of medicinal

Received: June 13, 2021

Accepted: August 18, 2021

Published: September 1, 2021 
Scheme 1. Pd-Catalyzed Direct Arylation of Donepezil with Aryl Bromides
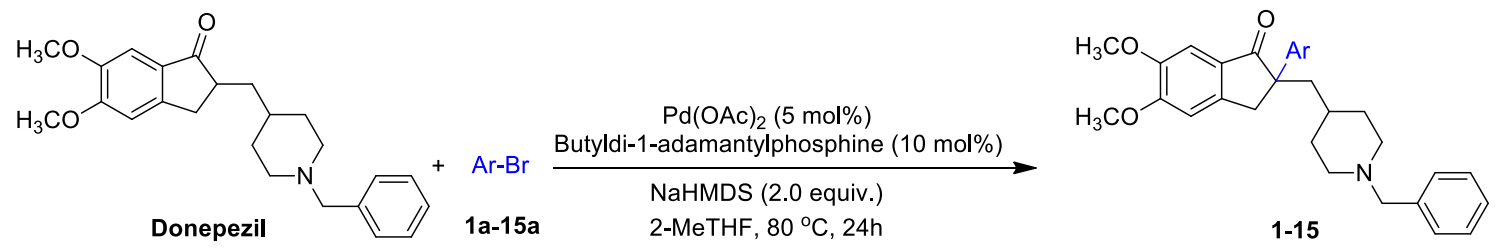

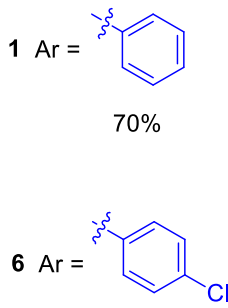

$55 \%$

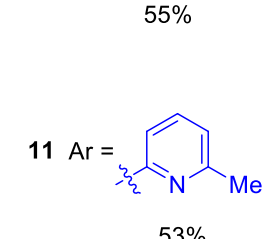

$53 \%$

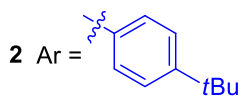

$77 \%$

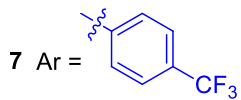

$56 \%$

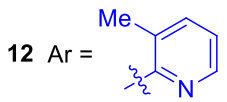

$60 \%$

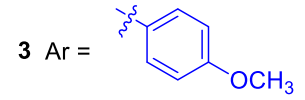

$74 \%$

$8 \mathrm{Ar}=$<smiles>Cc1cccc(C(F)(F)F)c1</smiles>

$54 \%$

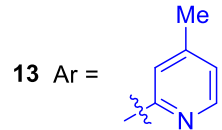

$65 \%$<smiles>CCOc1ccc([14C]2=[14CH][14CH]=[14CH][14CH]2[14CH2])cc1</smiles>

$68 \%$

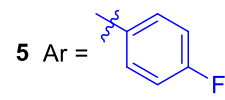

$59 \%$

$9 \mathrm{Ar}=$<smiles>CC(C)(N)c1cccc2ccccc12</smiles><smiles>COc1ccc2cc(C)ccc2c1</smiles>

$70 \%$

$67 \%$<smiles>[14CH3]c1ncccc1F</smiles><smiles></smiles>

$52 \%$

$59 \%$ chemistry, ${ }^{16-18}$ and so, we designed to directly install various aryl groups through palladium-catalyzed direct $\alpha$-arylation of ketone to give diverse aryldponepezils by the late-stage $\mathrm{C}-\mathrm{H}$ functionalization strategy.

All the previous modifications of donepezil were from the first intermediates, ${ }^{19,20}$ and there are no reports on the direct modification of donepezil, which should be a highly efficient way to provide donepezil derivatives. Palladium-catalyzed $\mathrm{C}-\mathrm{H}$ functionalization is a highly efficient method to form $\mathrm{C}-\mathrm{C}, \mathrm{C}-$ $\mathrm{N}$, or $\mathrm{C}-\mathrm{O}$ bonds, but it is a great challenge for the chemoselective $\mathrm{C}-\mathrm{H}$ functionalization in complex medicine molecules. On these bases, because the carbonyl oxygen of donepezil was involved in AChE binding, ${ }^{21}$ we proposed that the $\alpha$-arylated modification of the donepezil's ketone may lead to the great change of anti-AChE activity. Herein, we report the synthesis and bioevaluation of 15 new aryldonepezils (1-15).

\section{RESULTS AND DISCUSSION}

2.1. Chemistry. Initially, the reaction conditions of $\mathrm{Pd}$ catalyzed direct $\alpha$-arylation of the ketone in donepezil were optimized. According to the classic $\alpha$-arylation conditions, ${ }^{22}$ the different combinations of $\mathrm{Pd}(\mathrm{OAc})_{2}$, ligands (Tri-o-tolylphosphine, butyldi-1-adamantylphosphine, and MePhos), bases (NaOAc, $\mathrm{Cs}_{2} \mathrm{CO}_{3}, \mathrm{~K}_{2} \mathrm{O}$ tBu, and NaHMDS), and solvents [dioxane, tetrahydrofuran (THF), 2-MeTHF] were tested for the best yields. It was found that the $\mathrm{Pd}(\mathrm{OAc})_{2} /$ butyldi-1adamantylphosphine/NaHMDS-based catalytic system promoted the coupling of donepezil with 1-bromo-4-(tert-butyl)benzene (2a) in 2 -MeTHF at $80{ }^{\circ} \mathrm{C}$ for $24 \mathrm{~h}$ to furnish the desired compound (2) in $77 \%$ yield.

With the optimized reaction conditions in hand, the scope of the direct arylation of donepezil with aryl bromides was investigated (Scheme 1). In general, the phenyl bromides exhibited good selectivity and yield in the direct modification of donepezil. The phenyl bromides possessing the electrondonating groups $(t \mathrm{Bu}, \mathrm{OMe}$, and $\mathrm{OEt})$ resulted higher yields than those bearing the electron-withdrawing groups $(\mathrm{F}, \mathrm{Cl}$, and $\mathrm{CF}_{3}$ ). The arylation of donepezil with heterocyclic aryl bromides showed little lower yields than that of phenyl bromides (Scheme $1)$. The classic base-mediated deprotonative arylation mechanism is also shown in Figure 2. ${ }^{23}$

2.2. Biological Activities. 2.2.1. In Vitro Anti-cholinesterase Activity. The aryldonepezils (1-15) were evaluated in vitro for their anti-cholinesterase activity against $\mathrm{AChE}$ and $\mathrm{BuChE}$ in comparison to donepezil, and most compounds showed potential inhibition on AChE (Table 1). 3-Trifluoromethylphenyldonepezil (8) presented an almost similar inhibition rate with donepezil on AChE but showed only a $20.8 \%$ inhibition rate on $\mathrm{BuChE}$, which indicated that the trifluoromethyl group may play an important role in the anti-AChE activity of donepezil. In particular, the 3-methylpyridine and 4-methylpyridine derivates (12 and 13) displayed the more excellent inhibiting $\mathrm{AChE}$ activity than donepezil, but 6-methylpyridine derivate $\mathbf{1 1}$ showed low activity on AChE, suggesting the substituted position of pyridine may affect the anti-AChE activity of donepezil. Owing to the remarkable inhibition on both AChE and $\mathrm{BuChE}$, 3-methypridin-2-yl donepezil (12) needs an indepth investigation.

2.2.2. Cytotoxicity Evaluation. Because donepezil was approved for the $\mathrm{AD}$ treatment, the toxicity of aryldonepezils on SH-SY5Y cells was evaluated (Table 2). Most of aryldonepezils displayed low toxicity (3-13) except for compounds 1, 2, 14, and 15. Fortunately, the most potential $\mathrm{AChE}$ and BuChE inhibitors 8, 12, and 13 also exhibited significantly lower toxicity on SH-SY5Y cells than that of donepezil, suggesting the new donepezil-like anti-AD candidate medicines.

2.2.3. Neuroprotective Effects Against $\mathrm{H}_{2} \mathrm{O}_{2}$-Induced Cell Death in SH-SY5Y Cells. The neuroprotective activity of compounds 1-15 against $\mathrm{H}_{2} \mathrm{O}_{2}$-induced cell death in $\mathrm{SH}$ SY5Y cells was also evaluated in comparison to donepezil and all the donepezil derivates displayed protective activity on $\mathrm{H}_{2} \mathrm{O}_{2}$ - 


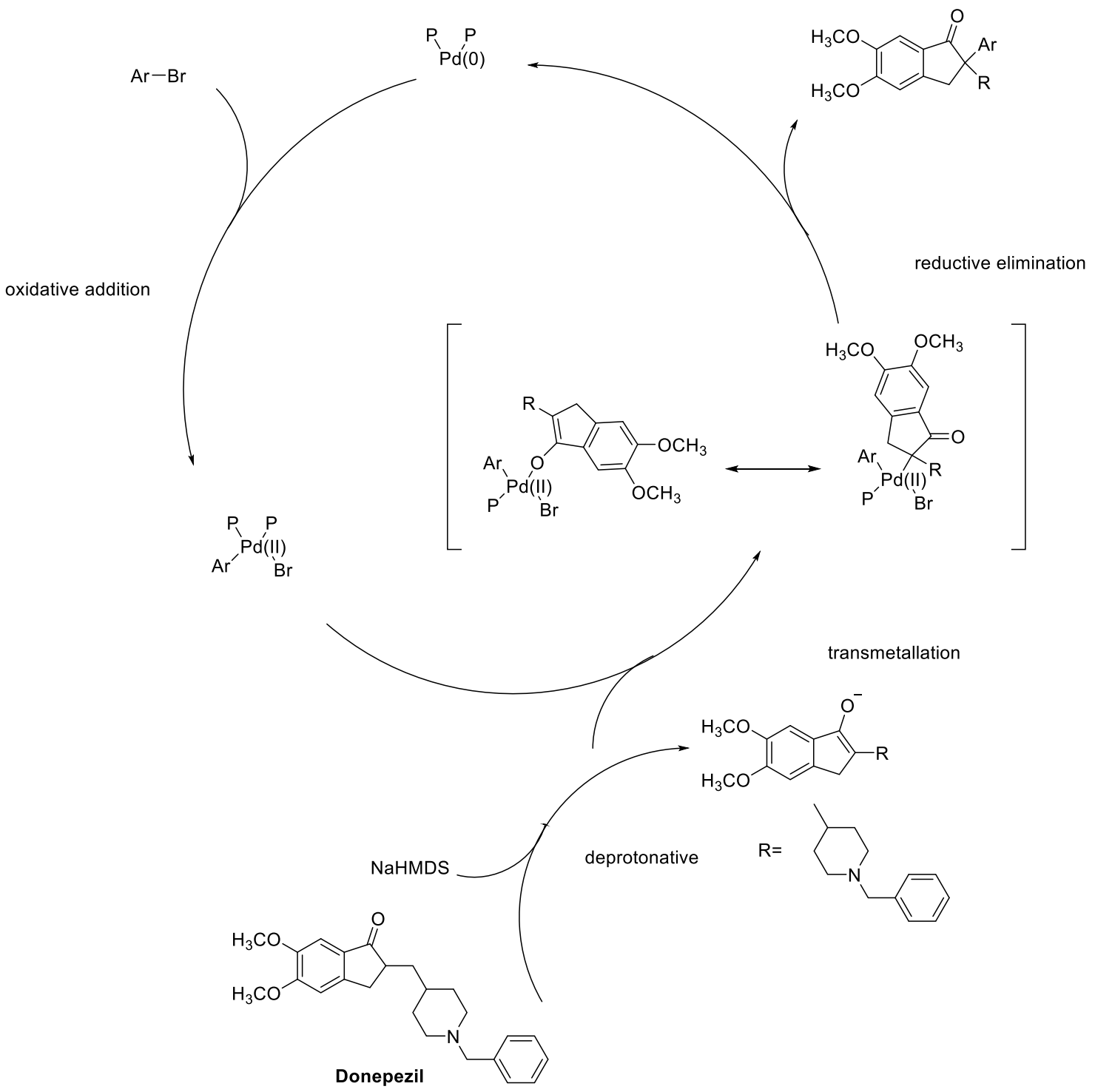

Figure 2. Catalytic cycle of the direct arylation of donepezil.

induced SH-SY5Y cell damage (Table 3). The survival rate of $\mathrm{H}_{2} \mathrm{O}_{2}(500 \mu \mathrm{M} / \mathrm{L})$-induced SH-SY5Y cells decreased to 28.47$87.87 \%$ after the following treatment of compounds $1-11$ and 13-15 but increased to $121.61 \%$ by the treatment of compound 12, suggesting the similar neuroprotective activity of compound 12 with donepezil.

2.3. Docking Studies. Because of the remarkable AChE inhibition activity, high neuroprotective activity, and low toxicity of pyridinyldonepezil (12), the molecular docking study with AChE of compound $\mathbf{1 2}$ was performed to interpret the possible binding mechanism.

Compound $\mathbf{1 2}$ does not interact directly with the catalytic triad (Ser200, Glu327, and His440). It has $\pi-\pi$ T-shaped interactions with aromatic amino acids of the active site and hydrophobic interactions. The benzene ring of indanone interacts with the indolic group of $\operatorname{Trp} 279$ via a $\pi-\pi$ T-shaped interaction. The piperidine ring created the $\pi$-alkyl interaction with Trp279 and created a carbon-hydrogen bond with Tyr70 (Figure 3). The PyRx software predicted that the $\mathrm{R}$ configuration is stronger than $\mathrm{S}$ configuration in binding to the site of AChE with the energy of -5.90 and $-4.83 \mathrm{kcal} / \mathrm{mol}$, respectively.
2.4. Blood-Brain Barrier Prediction. Penetrating the blood-brain barrier (BBB) is extremely significant for anti-AD medicines, and so, the BBB penetration of compounds 1-15 was predicted by using Calculate Molecular Properties (Discovery Studio 2020 Client). The predicted data are listed in Table 4. The value of level " 0 " showed that the brain-blood ratio is greater than $5: 1$.

\section{CONCLUSIONS}

In conclusion, 15 new aryldonepezil analogues were synthesized by Pd-catalyzed direct $\alpha$-arylation of ketone involving in the latestage $\mathrm{C}-\mathrm{H}$ functionalization strategy of donepezil. Bioevaluation of obtained compounds showed that 3-methylpridin-2-yl donepezil (12) exhibits remarkable inhibition against $\mathrm{AChE}$ and $\mathrm{BuChE}$, low toxicity on SH-SY5Y cells, and high neuroprotective activity compared with the parent compound donepezil, suggesting that it can be considered a very promising lead compound or medicine candidate for the treatment of $\mathrm{AD}$.

\section{EXPERIMENTAL SECTION}

4.1. Chemistry. 4.1.1. Materials and Methods. All reactions were conducted under an inert atmosphere of dry argon. 
Table 1. Inhibitory Activity Against AChE and BuChE by 1-15

\begin{tabular}{|c|c|c|c|c|}
\hline \multirow[b]{2}{*}{ compound } & \multicolumn{2}{|c|}{ AChE inhibition $[\%]^{a}$} & \multicolumn{2}{|c|}{$\mathrm{BuChE}$ inhibition [\%] ${ }^{a}$} \\
\hline & {$[\mathrm{I}]=100 \mu \mathrm{M}$} & {$[\mathrm{I}]=50 \mu \mathrm{M}$} & {$[\mathrm{I}]=100 \mu \mathrm{M}$} & {$[\mathrm{I}]=50 \mu \mathrm{M}$} \\
\hline 1 & $41.9 \pm 1.91$ & $19.6 \pm 2.67$ & $-44.7 \pm 3.11$ & $-65.0 \pm 2.33$ \\
\hline 2 & $34.1 \pm 0.20$ & $24.8 \pm 1.33$ & $-32.6 \pm 2.34$ & $-55.5 \pm 3.96$ \\
\hline 3 & $85.8 \pm 0.59$ & $86.7 \pm 0.03$ & $-34.2 \pm 4.22$ & $-23.5 \pm 2.34$ \\
\hline 4 & $68.6 \pm 3.65$ & $27.6 \pm 1.78$ & $47.2 \pm 2.01$ & $1.4 \pm 1.98$ \\
\hline 5 & $51.3 \pm 2.96$ & $48.7 \pm 2.53$ & $-76.8 \pm 2.12$ & $-100.9 \pm 2.05$ \\
\hline 6 & $71.2 \pm 1.52$ & $50.6 \pm 1.57$ & $-87.6 \pm 0.13$ & $-123.5 \pm 3.49$ \\
\hline 7 & $34.4 \pm 1.72$ & $20.8 \pm 3.49$ & $-11.2 \pm 0.99$ & $-156.1 \pm 1.21$ \\
\hline 8 & $101.1 \pm 2.87$ & $84.5 \pm 1.29$ & $20.8 \pm 3.09$ & $3.4 \pm 3.38$ \\
\hline 9 & $76.9 \pm 1.99$ & $49.0 \pm 1.52$ & $-19.6 \pm 3.05$ & $-60.1 \pm 2.09$ \\
\hline 10 & $42.6 \pm 3.76$ & $21.1 \pm 2.97$ & $-37.0 \pm 2.11$ & $-33.3 \pm 2.56$ \\
\hline 11 & $67.6 \pm 4.02$ & $59.9 \pm 3.12$ & $-12.6 \pm 2.89$ & $-76.0 \pm 4.44$ \\
\hline 12 & $105.1 \pm 2.96$ & $92.4 \pm 3.11$ & $88.9 \pm 3.33$ & $76.9 \pm 3.79$ \\
\hline 13 & $103.0 \pm 2.09$ & $79.9 \pm 2.11$ & $78.3 \pm 0.71$ & $56.8 \pm 2.56$ \\
\hline 14 & $30.9 \pm 0.08$ & $33.5 \pm 2.05$ & $-56.8 \pm 0.88$ & $-77.1 \pm 2.76$ \\
\hline 15 & $38.7 \pm 3.33$ & $23.31 \pm 2.09$ & $-44.5 \pm 1.54$ & $-34.8 \pm 1.13$ \\
\hline donepezil & $102.3 \pm 2.78$ & $88.0 \pm 1.21$ & $-10.39 \pm 0.74$ & $-20.1 \pm 3.23$ \\
\hline
\end{tabular}

${ }^{a} \mathrm{AChE}$ from Electrophorus electricus and BuChE from equine serum were used and percent inhibition data are the mean \pm SD of three independent experiments each performed in duplicate.

Table 2. Toxicity of Compounds 1-15 on SH-SY5Y Cells

\begin{tabular}{|c|c|c|c|c|c|}
\hline compound & survival rate (\%) & compound & survival rate (\%) & compound & survival rate (\%) \\
\hline 1 & $-0.15 \pm 0.77$ & 2 & $-1.91 \pm 1.09$ & 3 & $99.05 \pm 0.89$ \\
\hline 4 & $44.00 \pm 2.56$ & 5 & $9.40 \pm 1.54$ & 6 & $42.51 \pm 1.32$ \\
\hline 7 & $18.19 \pm 2.39$ & 8 & $147.42 \pm 1.43$ & 9 & $44.25 \pm 0.23$ \\
\hline 10 & $54.37 \pm 0.97$ & 11 & $73.58 \pm 2.39$ & 12 & $143.7 \pm 2.47$ \\
\hline 13 & $111.98 \pm 1.01$ & 14 & $-12.98 \pm 1.52$ & 15 & $-1.43 \pm 0.99$ \\
\hline donepezil & $102.71 \pm 0.39$ & & & & \\
\hline
\end{tabular}

Table 3. Neuroprotective Effects Against $\mathrm{H}_{2} \mathrm{O}_{2}$-Induced Cell Death in SH-SY5Y Cells

\begin{tabular}{|c|c|c|c|c|c|}
\hline compound & survival rate (\%) & compound & survival rate (\%) & compound & survival rate (\%) \\
\hline 1 & $55.25 \pm 1.11$ & 2 & $49.86 \pm 0.37$ & 3 & $42.90 \pm 1.98$ \\
\hline 4 & $62.24 \pm 2.59$ & 5 & $59.40 \pm 2.21$ & 6 & $51.08 \pm 2.94$ \\
\hline 7 & $50.59 \pm 2.41$ & 8 & $28.47 \pm 1.22$ & 9 & $53.23 \pm 3.33$ \\
\hline 10 & $39.01 \pm 0.99$ & 11 & $83.70 \pm 2.54$ & 12 & $121.61 \pm 3.98$ \\
\hline 13 & $87.87 \pm 3.67$ & 14 & $38.08 \pm 2.39$ & 15 & $14.00 \pm 2.76$ \\
\hline donepezil & $120.76 \pm 3.99$ & & & & \\
\hline
\end{tabular}
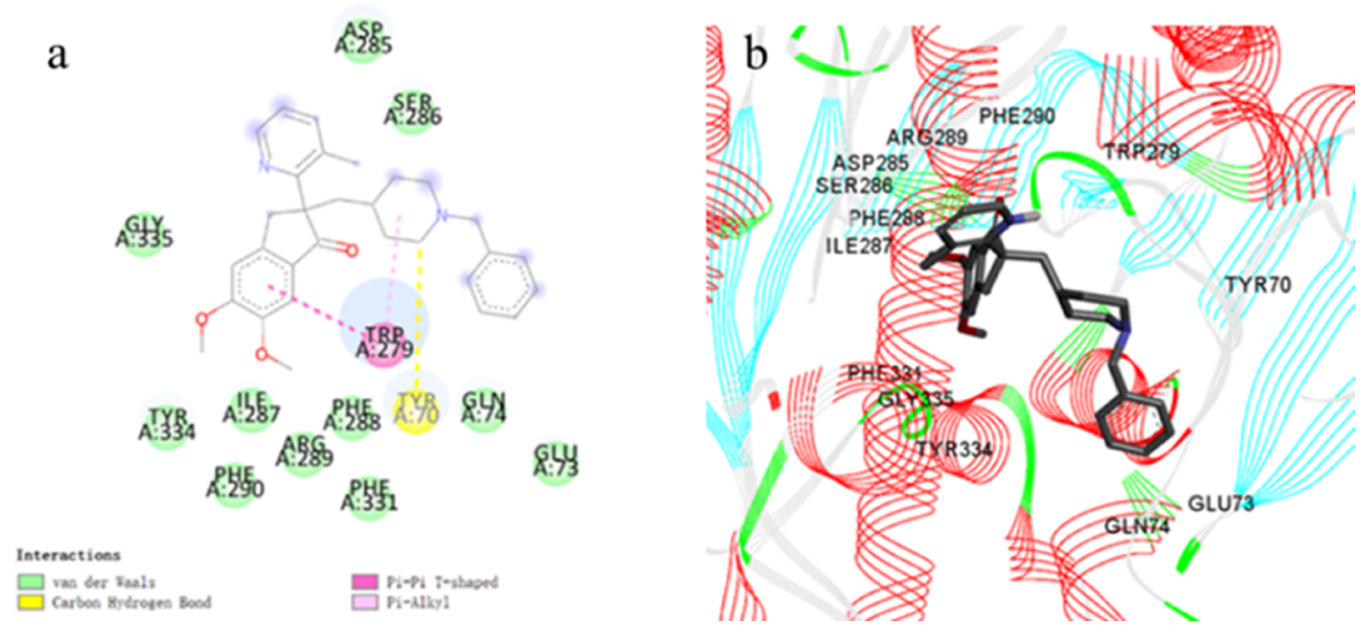

Figure 3. (a) Best pose of compound 12 in the active site of $\mathrm{AChE}$ and (b) residues of the active site involved in ligand binding. 
Table 4. BBB Prediction of Compounds by the Discovery Studio 2020 Client

compound
1
2
3
4
5
6
7
8

level
0
0
0
0
0
0
0
0

ADMET_BBB
1.097
0.98
0.95
1.058
1.16
1.302
1.201
1.102

compound
9
10
11
12
13
14
15
donepezil

BBB_LEVEL
0
0
0
0
0
0
0
0

ADMET_BBB
0.875
0.785
0.783
0.846
0.846
0.759
0.726
0.649

Anhydrous 2-MeTHF was purchased from Aladdin and used without further purification. Reactions were monitored by thin layer chromatography on silica gel plates (GF 254) using UV light to visualize the course of the reactions. Silica gel $\mathrm{H}$ (Qingdao Sea Chemical Factory, Qingdao, PR China) was used for column chromatography. NMR spectra were obtained using the Brüker $400 \mathrm{MHz}$ Fourier-transform NMR spectrometer at Chengdu Institute of Biology, Chinese Academy of Sciences NMR facility. Chemical shifts $(\delta)$ are reported in ppm with tetramethylsilane as the internal standard. Abbreviations for signal couplings are $\mathrm{s}$, singlet; $\mathrm{d}$, doublet; $\mathrm{t}$, triplet; and $\mathrm{m}$, multiplet. High-resolution mass spectrometry data were obtained on a Waters LC-TOF mass spectrometer (model LCT-XE Premier) using chemical ionization or electrospray ionization (ESI) in positive or negative mode depending on the analysis. Bromides were purchased from Aladdin and used as received.

4.1.2. General Synthesis of Compounds 1-15. The suspension of $\mathrm{Pd}(\mathrm{OAc})_{2}(5 \mathrm{~mol} \%)$ and butyldi-1-adamantylphosphine $(10 \mathrm{~mol} \%)$ in anhydrous 2 -MeTHF $(3.0 \mathrm{~mL})$ was stirred at $30^{\circ} \mathrm{C}$ under an argon atmosphere for $2 \mathrm{~h}$ to obtain a light pink solution. Then, the light pink solution was added to a $10 \mathrm{~mL}$ sealed dry reaction vial containing donepezil $(1.0 \mathrm{mmol})$, naphthalenyl, pyridinyl or quinolinyl bromides $(1.5 \mathrm{mmol})$, and NaHMDS $(2.0 \mathrm{mmol})$ via a syringe. The reaction mixture was stirred for $24 \mathrm{~h}$ at $80{ }^{\circ} \mathrm{C}$ before quenching with two drops of $\mathrm{H}_{2} \mathrm{O}$. The mixture was then diluted with ethyl acetate $(3 \mathrm{~mL})$ and filtered over a pad of $\mathrm{MgSO}_{4}$. The pad was rinsed with additional ethyl acetate, and the solution was concentrated in vacuo. The crude residue was loaded onto a silica gel column and purified by flash chromatography using petroleum etherEtOAc $(2: 1$ to $1: 2)$ as the eluent.

4.1.2.1. Compound 1. Yellow solid; yield 70\%; ${ }^{1} \mathrm{H}$ NMR $\left(400 \mathrm{MHz}\right.$, acetone- $\left.d_{6}\right): \delta 7.47(\mathrm{~d}, J=1.2 \mathrm{~Hz}, 1 \mathrm{H}), 7.45(\mathrm{~s}, 1 \mathrm{H})$, 7.35 (d, $J=6.8 \mathrm{~Hz}, 2 \mathrm{H}), 7.29$ (d, $J=7.2 \mathrm{~Hz}, 2 \mathrm{H}), 7.26$ (d, $J=8.1$ $\mathrm{Hz}, 2 \mathrm{H}), 7.24-7.16(\mathrm{~m}, 2 \mathrm{H}), 7.13(\mathrm{~s}, 1 \mathrm{H}), 7.06(\mathrm{~s}, 1 \mathrm{H}), 3.93(\mathrm{~s}$, $3 \mathrm{H}), 3.84(\mathrm{~s}, 3 \mathrm{H}), 3.63(\mathrm{~d}, J=17.2 \mathrm{~Hz}, 2 \mathrm{H}), 3.49(\mathrm{~s}, 2 \mathrm{H}), 3.36$ $(\mathrm{d}, J=17.2 \mathrm{~Hz}, 2 \mathrm{H}), 2.76(\mathrm{~s}, 2 \mathrm{H}), 2.24(\mathrm{dd}, J=14.2,3.8 \mathrm{~Hz}$, $2 \mathrm{H}), 1.88(\mathrm{dd}, J=14.2,6.5 \mathrm{~Hz}, 2 \mathrm{H}), 1.52(\mathrm{t}, J=12.0 \mathrm{~Hz}, 2 \mathrm{H})$, $1.38(\mathrm{~s}, 1 \mathrm{H}) .{ }^{13} \mathrm{C}$ NMR $\left(100 \mathrm{MHz}, \mathrm{CDCl}_{3}\right): \delta 206.4,155.9$, $149.8,147.8,142.7,138.3,129.5,128.6,128.6,128.3,127.1$, $126.7,126.6,107.2,105.3,63.5,57.5,56.4,56.2,53.8,53.8,44.8$, 41.0, 34.1, 33.4, 33.0. HR-ESI-MS: calcd for $\mathrm{C}_{30} \mathrm{H}_{34} \mathrm{NO}_{3}[\mathrm{M}+$ $\mathrm{H}]^{+}$, 456.2539; found, 456.2528.

4.1.2.2. Compound 2. Yellow solid; yield $77 \%$; ${ }^{1} \mathrm{H}$ NMR $\left(400 \mathrm{MHz}\right.$, acetone- $\left.d_{6}\right): \delta 7.40(\mathrm{~s}, 1 \mathrm{H}), 7.35(\mathrm{~d}, J=8.7 \mathrm{~Hz}, 2 \mathrm{H})$, $7.31(\mathrm{~d}, J=5.2 \mathrm{~Hz}, 2 \mathrm{H}), 7.30(\mathrm{~s}, 2 \mathrm{H}), 7.29-7.25(\mathrm{~m}, 2 \mathrm{H}), 7.13$ $(\mathrm{s}, 1 \mathrm{H}), 7.05(\mathrm{~s}, 1 \mathrm{H}), 3.93(\mathrm{~s}, 3 \mathrm{H}), 3.84(\mathrm{~s}, 3 \mathrm{H}), 3.61(\mathrm{~d}, J=17.2$ $\mathrm{Hz}, 2 \mathrm{H}), 3.53(\mathrm{~s}, 2 \mathrm{H}), 3.35$ (d, $J=17.2 \mathrm{~Hz}, 2 \mathrm{H}), 2.84(\mathrm{~s}, 2 \mathrm{H})$, $2.24(\mathrm{~m}, 2 \mathrm{H}), 1.92-1.79(\mathrm{~m}, 2 \mathrm{H}), 1.56(\mathrm{~s}, 2 \mathrm{H}), 1.45(\mathrm{~s}, 1 \mathrm{H})$, 1.25 (s, 9H). ${ }^{13} \mathrm{C}$ NMR (100 MHz, $\left.\mathrm{CDCl}_{3}\right): \delta$ 206.6, 156.0,
$149.8,149.5,147.9,139.5,130.3,128.7,128.7,128.2,126.3$, 125.6, 107.2, 105.2, 62.4, 57.0, 56.5, 56.3, 53.1, 44.3, 41.6, 34.5, 32.7, 32.2, 31.5. HR-ESI-MS: calcd for $\mathrm{C}_{34} \mathrm{H}_{42} \mathrm{NO}_{3}[\mathrm{M}+\mathrm{H}]^{+}$, 512.3165; found, 512.3149.

4.1.2.3. Compound 3. Yellow solid; yield $74 \%$; ${ }^{1} \mathrm{H}$ NMR $\left(400 \mathrm{MHz}\right.$, acetone- $\left.d_{6}\right): \delta 7.43(\mathrm{~s}, 1 \mathrm{H}), 7.38-7.35(\mathrm{~m}, 2 \mathrm{H})$, $7.31(\mathrm{~d}, J=6.7 \mathrm{~Hz}, 2 \mathrm{H}), 7.29-7.24(\mathrm{~m}, 2 \mathrm{H}), 7.12(\mathrm{~s}, 1 \mathrm{H}), 7.05$ $(\mathrm{s}, 1 \mathrm{H}), 6.84-6.80(\mathrm{~m}, 2 \mathrm{H}), 3.93(\mathrm{~s}, 3 \mathrm{H}), 3.83(\mathrm{~s}, 3 \mathrm{H}), 3.73(\mathrm{~s}$, $3 \mathrm{H}), 3.63$ (s, 2H), 3.59 (s, $2 \mathrm{H}), 3.32$ (d, J=17.2 Hz, $2 \mathrm{H}), 2.84$ $(\mathrm{s}, 2 \mathrm{H}), 2.22(\mathrm{~d}, J=14.3 \mathrm{~Hz}, 2 \mathrm{H}), 1.83(\mathrm{~d}, J=11.5 \mathrm{~Hz}, 2 \mathrm{H}), 1.54$ $(\mathrm{d}, J=15.6 \mathrm{~Hz}, 2 \mathrm{H}), 1.44(\mathrm{~s}, 1 \mathrm{H}) .{ }^{13} \mathrm{C} \mathrm{NMR}(150 \mathrm{MHz}$, $\left.\mathrm{CDCl}_{3}\right): \delta 206.6,158.3,155.9,149.8,147.7,134.6,129.5,128.6$, $128.3,127.8,127.2,114.0,107.2,105.4,63.5,56.9,56.4,56.3$, 55.4, 53.9, 44.9, 41.0, 34.1, 33.4, 33.1, 32.1. HR-ESI-MS: calcd for $\mathrm{C}_{31} \mathrm{H}_{36} \mathrm{NO}_{4}[\mathrm{M}+\mathrm{H}]^{+}$, 486.2644; found, 486.2635 .

4.1.2.4. Compound 4. Yellow solid; yield $68 \%$; ${ }^{1} \mathrm{H}$ NMR $\left(600 \mathrm{MHz}, \mathrm{CDCl}_{3}\right): \delta 7.29(\mathrm{~d}, J=8.5 \mathrm{~Hz}, 2 \mathrm{H}), 7.28-7.25(\mathrm{~m}$, $4 \mathrm{H}), 7.22(\mathrm{~d}, J=6.6 \mathrm{~Hz}, 1 \mathrm{H}), 7.15(\mathrm{~s}, 1 \mathrm{H}), 6.89(\mathrm{~s}, 1 \mathrm{H}), 6.78(\mathrm{~d}$, $J=8.6 \mathrm{~Hz}, 2 \mathrm{H}), 3.97(\mathrm{~s}, 3 \mathrm{H}), 3.88(\mathrm{~s}, 3 \mathrm{H}), 3.53(\mathrm{~d}, J=17.0 \mathrm{~Hz}$, $1 \mathrm{H}), 3.41(\mathrm{~s}, 2 \mathrm{H}), 3.28(\mathrm{~d}, J=17.0 \mathrm{~Hz}, 1 \mathrm{H}), 2.74(\mathrm{~s}, 2 \mathrm{H}), 2.24-$ $2.13(\mathrm{~m}, 2 \mathrm{H}), 1.90-1.84(\mathrm{~m}, 2 \mathrm{H}), 1.82-1.74(\mathrm{~m}, 2 \mathrm{H}), 1.49-$ $1.42(\mathrm{~m}, 2 \mathrm{H}), 1.37(\mathrm{~d}, J=6.9 \mathrm{~Hz}, 3 \mathrm{H}), 1.31(\mathrm{~d}, J=3.1 \mathrm{~Hz}, 2 \mathrm{H})$, $0.88(\mathrm{t}, J=6.8 \mathrm{~Hz}, 1 \mathrm{H}) .{ }^{13} \mathrm{C} \mathrm{NMR}\left(150 \mathrm{MHz}, \mathrm{CDCl}_{3}\right): \delta 206.6$, $157.7,155.9,149.8,147.7,138.5,134.4,129.5,128.6,128.3$, $127.8,127.1,114.5,107.2,105.4,63.6,63.6,56.9,56.4,56.3$, 53.9, 53.9, 44.9, 40.9, 34.1, 33.5, 33.1, 15.0. HR-ESI-MS: calcd for $\mathrm{C}_{32} \mathrm{H}_{38} \mathrm{NO}_{4}[\mathrm{M}+\mathrm{H}]^{+}$, 500.2801; found, 500.2791.

4.1.2.5. Compound 5. Yellow solid; yield 59\%; ${ }^{1} \mathrm{H}$ NMR $\left(400 \mathrm{MHz}\right.$, acetone- $\left.d_{6}\right): \delta 7.45(\mathrm{~d}, J=7.6 \mathrm{~Hz}, 2 \mathrm{H}), 7.34-7.29$ $(\mathrm{m}, 2 \mathrm{H}), 7.28(\mathrm{~s}, 2 \mathrm{H}), 7.25(\mathrm{~d}, J=7.8 \mathrm{~Hz}, 2 \mathrm{H}), 7.17(\mathrm{~d}, J=7.4$ $\mathrm{Hz}, 1 \mathrm{H}), 7.14$ (s, 1H), 7.06 (s, 1H), 3.94 (s, 3H), $3.84(\mathrm{~s}, 3 \mathrm{H})$, $3.64(\mathrm{~d}, J=17.2 \mathrm{~Hz}, 2 \mathrm{H}), 3.45(\mathrm{~s}, 2 \mathrm{H}), 3.36(\mathrm{~d}, J=17.2 \mathrm{~Hz}, 2 \mathrm{H})$, $2.74(\mathrm{~s}, 2 \mathrm{H}), 2.31-2.19(\mathrm{~m}, 2 \mathrm{H}), 1.94-1.85(\mathrm{~m}, 2 \mathrm{H}), 1.51(\mathrm{t}, J$ $=13.5 \mathrm{~Hz}, 2 \mathrm{H}), 1.36(\mathrm{~s}, 1 \mathrm{H}) \cdot{ }^{13} \mathrm{C}$ NMR $\left(100 \mathrm{MHz}, \mathrm{CDCl}_{3}\right): \delta$ 206.3, 156.0, 148.8 (d, $J=220.0 \mathrm{~Hz}), 142.7,129.9,128.7,128.5$, 127.8, 126.7, 107.2, 105.3, 63.0, 57.4, 56.5, 56.3, 53.5, 44.8, 41.4, 33.3, 32.6, 32.1, 30.8. HR-ESI-MS: calcd for $\mathrm{C}_{30} \mathrm{H}_{33} \mathrm{FNO}_{3}[\mathrm{M}+$ $\mathrm{H}]^{+}$, 474.2444; found, 474.2430.

4.1.2.6. Compound 6. Yellow solid; yield 55\%; ${ }^{1} \mathrm{H}$ NMR $\left(400 \mathrm{MHz}\right.$, acetone- $\left.d_{6}\right): \delta 7.49-7.46(\mathrm{~m}, 2 \mathrm{H}), 7.43(\mathrm{~d}, J=7.1$ $\mathrm{Hz}, 2 \mathrm{H}), 7.31(\mathrm{~d}, J=6.6 \mathrm{~Hz}, 2 \mathrm{H}), 7.28(\mathrm{~d}, J=2.9 \mathrm{~Hz}, 2 \mathrm{H}), 7.26$ $(\mathrm{d}, J=2.9 \mathrm{~Hz}, 1 \mathrm{H}), 7.14(\mathrm{~s}, 1 \mathrm{H}), 7.06(\mathrm{~s}, 1 \mathrm{H}), 3.94(\mathrm{~s}, 3 \mathrm{H}), 3.84$ (s, $3 \mathrm{H}), 3.63(\mathrm{~d}, J=5.9 \mathrm{~Hz}, 2 \mathrm{H}), 3.59$ (d, $J=6.5 \mathrm{~Hz}, 2 \mathrm{H}), 3.37$ (d, $J=17.2 \mathrm{~Hz}, 2 \mathrm{H}), 2.89-2.83(\mathrm{~m}, 2 \mathrm{H}), 2.21(\mathrm{dd}, J=14.2,3.0$ $\mathrm{Hz}, 2 \mathrm{H}), 1.87$ (dd, $J=14.2,5.8 \mathrm{~Hz}, 2 \mathrm{H}), 1.53(\mathrm{~d}, J=9.2 \mathrm{~Hz}$, $2 \mathrm{H}), 1.44(\mathrm{~s}, 1 \mathrm{H}) .{ }^{13} \mathrm{C} \mathrm{NMR}\left(100 \mathrm{MHz}, \mathrm{CDCl}_{3}\right): \delta 205.7$, $156.2,150.0,147.4,141.0,132.6,130.2,129.5,128.7,128.7$, $128.4,128.2,128.2,107.2,105.2,62.5,56.8,56.5,56.3,53.2$, 53.1, 44.8, 41.5, 32.7, 32.2, 32.0. HR-ESI-MS: calcd for $\mathrm{C}_{30} \mathrm{H}_{33} \mathrm{ClNO}_{3}[\mathrm{M}+\mathrm{H}]^{+}$, 490.2149; found, 490.2130. 
4.1.2.7. Compound 7. Yellow solid; yield 56\%; ${ }^{1} \mathrm{H}$ NMR $\left(400 \mathrm{MHz}\right.$, acetone- $\left.d_{6}\right): \delta 7.70(\mathrm{~d}, J=8.5 \mathrm{~Hz}, 2 \mathrm{H}), 7.62(\mathrm{~d}, J=$ $8.5 \mathrm{~Hz}, 2 \mathrm{H}), 7.50(\mathrm{~s}, 2 \mathrm{H}), 7.33(\mathrm{~d}, J=5.8 \mathrm{~Hz}, 1 \mathrm{H}), 7.30(\mathrm{~d}, J=$ $6.1 \mathrm{~Hz}, 2 \mathrm{H}), 7.16(\mathrm{~s}, 1 \mathrm{H}), 7.07(\mathrm{~s}, 1 \mathrm{H}), 3.94(\mathrm{~s}, 3 \mathrm{H}), 3.85(\mathrm{~s}$, $3 \mathrm{H}), 3.74(\mathrm{~s}, 2 \mathrm{H}), 3.67(\mathrm{~d}, J=17.4 \mathrm{~Hz}, 2 \mathrm{H}), 3.43(\mathrm{~d}, J=17.4 \mathrm{~Hz}$, $2 \mathrm{H}), 2.93(\mathrm{~s}, 2 \mathrm{H}), 2.31-2.25(\mathrm{~m}, 2 \mathrm{H}), 1.95(\mathrm{dd}, J=14.4,6.5 \mathrm{~Hz}$, $2 \mathrm{H}), 1.70(\mathrm{~s}, 1 \mathrm{H}), 1.58(\mathrm{~s}, 2 \mathrm{H}) .{ }^{13} \mathrm{C} \mathrm{NMR}\left(100 \mathrm{MHz} \mathrm{CDCl}_{3}\right): \delta$ $205.3,156.4,148.8$ (q, $J=270.0 \mathrm{~Hz}), 146.6,130.5,129.2,128.9$, 128.7, 128.2, 127.1, 125.6, 125.6, 107.2, 105.1, 62.1, 57.1, 56.5, 56.3, 52.9, 52.8, 44.7, 41.8, 32.1, 31.9, 31.5. HR-ESI-MS: calcd for $\mathrm{C}_{31} \mathrm{H}_{33} \mathrm{~F}_{3} \mathrm{NO}_{3}[\mathrm{M}+\mathrm{H}]^{+}$, 524.2413; found, 524.2400.

4.1.2.8. Compound 8. Yellow solid; yield 54\%; ${ }^{1} \mathrm{H}$ NMR $\left(400 \mathrm{MHz}\right.$, acetone- $\left.d_{6}\right): \delta 7.85(\mathrm{~s}, 1 \mathrm{H}), 7.80-7.76(\mathrm{~m}, 1 \mathrm{H})$, $7.54(\mathrm{~s}, 1 \mathrm{H}), 7.52(\mathrm{~s}, 1 \mathrm{H}), 7.27(\mathrm{~s}, 2 \mathrm{H}), 7.25(\mathrm{~s}, 2 \mathrm{H}), 7.22-7.18$ (m, 1H), $7.15(\mathrm{~s}, 1 \mathrm{H}), 7.09(\mathrm{~s}, 1 \mathrm{H}), 3.94(\mathrm{~s}, 3 \mathrm{H}), 3.85(\mathrm{~s}, 3 \mathrm{H})$, $3.69(\mathrm{~d}, J=17.4 \mathrm{~Hz}, 1 \mathrm{H}), 3.46(\mathrm{~d}, J=17.4 \mathrm{~Hz}, 1 \mathrm{H}), 3.35(\mathrm{~s}, 2 \mathrm{H})$, $2.73-2.65(\mathrm{~m}, 2 \mathrm{H}), 2.22(\mathrm{dd}, J=14.2,4.4 \mathrm{~Hz}, 1 \mathrm{H}), 1.97-1.89$ $(\mathrm{m}, 1 \mathrm{H}), 1.81-1.67(\mathrm{~m}, 2 \mathrm{H}), 1.45(\mathrm{~m}, J=15.1,9.4,2.7 \mathrm{~Hz}, 2 \mathrm{H})$, $1.38-1.25(\mathrm{~m}, 2 \mathrm{H}), 1.22(\mathrm{dd}, J=7.5,4.1 \mathrm{~Hz}, 1 \mathrm{H}) .{ }^{13} \mathrm{C} \mathrm{NMR}$ $\left(100 \mathrm{MHz}, \mathrm{CDCl}_{3}\right): \delta 205.6,156.2,148.8(\mathrm{q}, J=250.0 \mathrm{~Hz})$ $144.0,138.2$, 131.0, 130.7, 130.3, 129.4, 129.0, 128.4, 128.3, $127.2,123.5,107.2,105.2,63.4,57.2,56.5,56.3,53.8,53.7,45.4$, 40.8, 34.0, 33.4, 33.0, 29.8. HR-ESI-MS: calcd for $\mathrm{C}_{31} \mathrm{H}_{33} \mathrm{~F}_{3} \mathrm{NO}_{3}$ $[\mathrm{M}+\mathrm{H}]^{+}$, 524.2413; found, 524.2401.

4.1.2.9. Compound 9. Yellow solid; yield 70\%; ${ }^{1} \mathrm{H}$ NMR $\left(600 \mathrm{MHz}, \mathrm{CDCl}_{3}\right): \delta 7.94(\mathrm{~d}, J=7.9 \mathrm{~Hz}, 1 \mathrm{H}), 7.83(\mathrm{t}, J=9.9$ $\mathrm{Hz}, 2 \mathrm{H}), 7.71(\mathrm{~d}, J=8.1 \mathrm{~Hz}, 1 \mathrm{H}), 7.56(\mathrm{~d}, J=7.4 \mathrm{~Hz}, 1 \mathrm{H}), 7.45-$ $7.40(\mathrm{~m}, 2 \mathrm{H}), 7.34(\mathrm{~s}, 1 \mathrm{H}), 7.32(\mathrm{~s}, 1 \mathrm{H}), 7.28(\mathrm{~d}, J=7.6 \mathrm{~Hz}$, $3 \mathrm{H}), 7.24-7.21(\mathrm{~m}, 1 \mathrm{H}), 6.82(\mathrm{~s}, 1 \mathrm{H}), 3.97(\mathrm{~s}, 3 \mathrm{H}), 3.94(\mathrm{~s}$, $3 \mathrm{H}), 3.70-3.61(\mathrm{~m}, 2 \mathrm{H}), 3.46(\mathrm{~d}, J=38.2 \mathrm{~Hz}, 2 \mathrm{H}), 2.78-2.67$ (m, 2H), 2.46-2.34 (m, 2H), 2.27 (dd, $J=14.5,4.5 \mathrm{~Hz}, 2 \mathrm{H})$, $1.91(\mathrm{~s}, 2 \mathrm{H}), 1.78$ (s, 2H), 0.85 (s, 1H). ${ }^{13} \mathrm{C}$ NMR (100 MHz, $\left.\mathrm{CDCl}_{3}\right): \delta 207.7,156.2,150.0,147.6,140.0,135.3,131.5,130.0$, $129.6,129.4,128.4,128.4,128.3,127.4,127.2,125.8,125.4$, 125.3, 125.2, 107.4, 105.0, 63.3, 58.0, 56.4, 56.3, 53.8, 53.7, 44.9, 42.7, 34.2, 33.3, 32.7. HR-ESI-MS: calcd for $\mathrm{C}_{34} \mathrm{H}_{36} \mathrm{NO}_{3}[\mathrm{M}+$ $\mathrm{H}]^{+}$, 506.2695; found, 506.2682.

4.1.2.10. Compound 10. Yellow solid; yield $67 \%$; ${ }^{1} \mathrm{H}$ NMR $\left(400 \mathrm{MHz}\right.$, acetone- $\left.d_{6}\right): \delta 7.86(\mathrm{~s}, 1 \mathrm{H}), 7.73(\mathrm{~s}, 1 \mathrm{H}), 7.71(\mathrm{~s}$, $1 \mathrm{H}), 7.59-7.56(\mathrm{~m}, 1 \mathrm{H}), 7.57-7.53(\mathrm{~m}, 1 \mathrm{H}), 7.32(\mathrm{~s}, 2 \mathrm{H})$, $7.31-7.26(\mathrm{~m}, 2 \mathrm{H}), 7.22(\mathrm{~d}, J=2.3 \mathrm{~Hz}, 1 \mathrm{H}), 7.15(\mathrm{~s}, 1 \mathrm{H}), 7.10-$ $7.07(\mathrm{~m}, 1 \mathrm{H}), 7.07(\mathrm{~s}, 1 \mathrm{H}), 3.94(\mathrm{~s}, 3 \mathrm{H}), 3.87(\mathrm{~s}, 3 \mathrm{H}), 3.82(\mathrm{~s}$, $3 \mathrm{H}), 3.73(\mathrm{~d}, J=17.3 \mathrm{~Hz}, 1 \mathrm{H}), 3.37(\mathrm{~d}, J=17.3 \mathrm{~Hz}, 1 \mathrm{H}), 2.96(\mathrm{~s}$, $2 \mathrm{H}), 2.34(\mathrm{dd}, J=14.3,3.9 \mathrm{~Hz}, 2 \mathrm{H}), 2.28-2.12(\mathrm{~m}, 1 \mathrm{H}), 2.09(\mathrm{~s}$, $3 \mathrm{H}), 1.95$ (dd, $J=14.0,7.1 \mathrm{~Hz}, 1 \mathrm{H}), 1.62(\mathrm{~s}, 4 \mathrm{H}) .{ }^{13} \mathrm{C}$ NMR $\left(150 \mathrm{MHz}, \mathrm{CDCl}_{3}\right): \delta 206.4,157.9,156.04,149.9,147.7,137.7$, $133.4,129.8,129.7,128.9,128.8,128.4,127.5,127.3,125.9$, 125.0, 123.5, 119.0, 107.3, 105.6, 105.4, 63.2, 57.4, 56.5, 56.3, 55.5, 53.6, 44.9, 41.3, 33.7, 32.9, 32.2, 31.9. HR-ESI-MS: calcd for $\mathrm{C}_{35} \mathrm{H}_{38} \mathrm{NO}_{4}[\mathrm{M}+\mathrm{H}]^{+}$, 536.2801; found, 536.2791.

4.1.2.11. Compound 11. Yellow solid; yield 53\%; ${ }^{1} \mathrm{H}$ NMR $\left(600 \mathrm{MHz}, \mathrm{CDCl}_{3}\right): \delta 7.47(\mathrm{q}, J=5.1,4.5 \mathrm{~Hz}, 2 \mathrm{H}), 7.26(\mathrm{q}, J=$ $6.9 \mathrm{~Hz}, 5 \mathrm{H}), 7.13(\mathrm{~s}, 1 \mathrm{H}), 6.95-6.89(\mathrm{~m}, 2 \mathrm{H}), 4.40$ (d, $J=17.0$ $\mathrm{Hz}, 1 \mathrm{H}), 3.98(\mathrm{~s}, 3 \mathrm{H}), 3.88(\mathrm{~s}, 3 \mathrm{H}), 3.45-3.37(\mathrm{~m}, 2 \mathrm{H}), 3.13(\mathrm{~d}$, $J=17.0 \mathrm{~Hz}, 1 \mathrm{H}), 2.73(\mathrm{t}, J=13.6 \mathrm{~Hz}, 2 \mathrm{H}), 2.45(\mathrm{~s}, 3 \mathrm{H}), 2.25-$ $2.15(\mathrm{~m}, 1 \mathrm{H}), 1.93(\mathrm{dd}, J=14.4,5.5 \mathrm{~Hz}, 1 \mathrm{H}), 1.84-1.68(\mathrm{~m}$, $4 \mathrm{H}), 1.56-1.34(\mathrm{~m}, 2 \mathrm{H}), 0.87(\mathrm{dd}, J=18.2,11.3 \mathrm{~Hz}, 1 \mathrm{H}) .{ }^{13} \mathrm{C}$ NMR $\left(150 \mathrm{MHz}, \mathrm{CDCl}_{3}\right): \delta 206.7,160.0,157.3,156.0,149.6$, $149.5,136.6,129.5,128.5,128.3,127.1,121.2,119.0,107.5$, 105.0, 63.6, 60.3, 56.4, 56.3, 53.9, 45.6, 37.7, 33.8, 33.4, 29.9, 24.8. HR-ESI-MS: calcd for $\mathrm{C}_{30} \mathrm{H}_{35} \mathrm{~N}_{2} \mathrm{O}_{3}[\mathrm{M}+\mathrm{H}]^{+}, 471.2648$; found, 471.2630 .
4.1.2.12. Compound 12. Yellow solid; yield $60 \% ;{ }^{1} \mathrm{H}$ NMR $\left(400 \mathrm{MHz}\right.$, acetone- $\left.d_{6}\right): \delta 8.36(\mathrm{~d}, J=3.3 \mathrm{~Hz}, 1 \mathrm{H}), 7.62(\mathrm{~s}, 1 \mathrm{H})$, $7.49(\mathrm{~d}, J=7.2 \mathrm{~Hz}, 1 \mathrm{H}), 7.35(\mathrm{~d}, J=8.9 \mathrm{~Hz}, 2 \mathrm{H}), 7.32(\mathrm{~d}, J=1.6$ $\mathrm{Hz}, 2 \mathrm{H}), 7.17(\mathrm{~s}, 1 \mathrm{H}), 7.16-7.13(\mathrm{~m}, 1 \mathrm{H}), 7.11(\mathrm{~s}, 1 \mathrm{H}), 3.93(\mathrm{~s}$, $3 \mathrm{H}), 3.90-3.89(\mathrm{~m}, 1 \mathrm{H}), 3.87(\mathrm{~s}, 3 \mathrm{H}), 3.35(\mathrm{~d}, J=17.2 \mathrm{~Hz}, 1 \mathrm{H})$, $3.12(\mathrm{~d}, J=17.3 \mathrm{~Hz}, 1 \mathrm{H}), 3.00(\mathrm{~s}, 2 \mathrm{H}), 2.63(\mathrm{dd}, J=14.2,4.1 \mathrm{~Hz}$, $1 \mathrm{H}), 2.44-2.23(\mathrm{~m}, 2 \mathrm{H}), 1.95(\mathrm{~s}, 3 \mathrm{H}), 1.89(\mathrm{~d}, J=13.0 \mathrm{~Hz}, 1 \mathrm{H})$, $1.77(\mathrm{~d}, J=14.3 \mathrm{~Hz}, 2 \mathrm{H}), 1.64(\mathrm{~s}, 2 \mathrm{H}), 1.56(\mathrm{~s}, 2 \mathrm{H}) .{ }^{13} \mathrm{C} \mathrm{NMR}$ $\left(100 \mathrm{MHz}_{\mathrm{CDCl}}\right): \delta 206.7,159.4,155.8,150.0,147.2,145.8$, $139.7,132.0,130.4,129.7,129.5,129.4,128.3,122.1,107.5$, 104.8, 60.9, 56.5, 56.3, 53.7, 53.4, 53.3, 43.8, 42.5, 33.6, 32.8, 32.3, 20.1. HR-ESI-MS: calcd for $\mathrm{C}_{30} \mathrm{H}_{35} \mathrm{~N}_{2} \mathrm{O}_{3}[\mathrm{M}+\mathrm{H}]^{+}$, 471.2648; found, 471.2634 .

4.1.2.13. Compound 13. Yellow solid; yield $65 \%$; ${ }^{1} \mathrm{H}$ NMR $\left(600 \mathrm{MHz}, \mathrm{CDCl}_{3}\right): \delta 8.31(\mathrm{~d}, J=4.7 \mathrm{~Hz}, 1 \mathrm{H}), 7.50(\mathrm{~s}, 1 \mathrm{H})$, $7.33-7.29(\mathrm{~m}, 1 \mathrm{H}), 7.26(\mathrm{~d}, J=5.1 \mathrm{~Hz}, 2 \mathrm{H}), 7.25(\mathrm{~s}, 1 \mathrm{H}), 7.22$ $(\mathrm{d}, J=6.9 \mathrm{~Hz}, 1 \mathrm{H}), 7.13(\mathrm{~s}, 1 \mathrm{H}), 6.94-6.84(\mathrm{~m}, 2 \mathrm{H}), 4.28(\mathrm{~d}, J=$ $17.0 \mathrm{~Hz}, 1 \mathrm{H}), 3.96(\mathrm{~d}, J=7.6 \mathrm{~Hz}, 3 \mathrm{H}), 3.89$ (d, J=14.8 Hz, $3 \mathrm{H})$, $3.51(\mathrm{~s}, 1 \mathrm{H}), 3.41(\mathrm{~s}, 2 \mathrm{H}), 3.16(\mathrm{~d}, J=17.0 \mathrm{~Hz}, 1 \mathrm{H}), 2.77-2.65$ $(\mathrm{m}, 2 \mathrm{H}), 2.31(\mathrm{~s}, 3 \mathrm{H}), 2.26-2.15(\mathrm{~m}, 1 \mathrm{H}), 1.96-1.90(\mathrm{~m}, 2 \mathrm{H})$, $1.83-1.74(\mathrm{~m}, 2 \mathrm{H}), 1.69-1.46(\mathrm{~m}, 2 \mathrm{H}), 0.93-0.74(\mathrm{~m}, 1 \mathrm{H})$. ${ }^{13} \mathrm{C}$ NMR $\left(150 \mathrm{MHz} \mathrm{CDCl}_{3}\right): \delta 206.5,160.8,156.0,149.7$, $149.3,148.5,147.6,129.5,128.5,128.4,128.3,127.1,123.1$, $122.9,107.5,105.0,63.6,60.1,56.4,56.2,53.9,45.7,45.4,37.9$, 34.7, 33.8, 33.6, 33.4. HR-ESI-MS: calcd for $\mathrm{C}_{30} \mathrm{H}_{35} \mathrm{~N}_{2} \mathrm{O}_{3}[\mathrm{M}+$ $\mathrm{H}]^{+}$, 471.2648; found, 471.2636.

4.1.2.14. Compound 14. Yellow solid; yield 52\%; ${ }^{1} \mathrm{H}$ NMR $\left(601 \mathrm{MHz}, \mathrm{CDCl}_{3}\right): \delta 7.47(\mathrm{~d}, J=6.5 \mathrm{~Hz}, 2 \mathrm{H}), 7.26(\mathrm{~d}, J=9.8$ $\mathrm{Hz}, 4 \mathrm{H}), 7.24-7.20(\mathrm{~m}, 1 \mathrm{H}), 7.13(\mathrm{~s}, 1 \mathrm{H}), 6.95-6.89(\mathrm{~m}, 2 \mathrm{H})$, $4.40(\mathrm{~d}, J=17.0 \mathrm{~Hz}, 1 \mathrm{H}), 3.97(\mathrm{~s}, 3 \mathrm{H}), 3.88(\mathrm{~s}, 3 \mathrm{H}), 3.45-3.37$ $(\mathrm{m}, 2 \mathrm{H}), 3.13(\mathrm{~d}, J=17.1 \mathrm{~Hz}, 1 \mathrm{H}), 2.73(\mathrm{t}, J=13.6 \mathrm{~Hz}, 2 \mathrm{H})$, $2.45(\mathrm{~s}, 2 \mathrm{H}), 2.25-2.16(\mathrm{~m}, 1 \mathrm{H}), 1.92(\mathrm{dt}, J=16.8,8.1 \mathrm{~Hz}, 2 \mathrm{H})$, $1.84-1.73(\mathrm{~m}, 2 \mathrm{H}), 1.58-1.45(\mathrm{~m}, 1 \mathrm{H}), 0.87(\mathrm{dd}, J=17.9,10.9$ $\mathrm{Hz}, 1 \mathrm{H}) .{ }^{13} \mathrm{C}$ NMR $\left(150 \mathrm{MHz}, \mathrm{CDCl}_{3}\right): \delta 206.7,160.0,156.6$ $(\mathrm{d}, J=180.0 \mathrm{~Hz}), 149.6,149.5,138.5,136.5,129.5,128.5,128.3$, 127.1, 121.2, 119.0, 107.5, 105.0, 63.6, 60.3, 56.4, 56.2, 53.9, 45.6, 37.7, 33.8, 33.4, 30.4, 24.8. HR-ESI-MS: calcd for $\mathrm{C}_{29} \mathrm{H}_{32} \mathrm{FN}_{2} \mathrm{O}_{3}[\mathrm{M}+\mathrm{H}]^{+}$, 475.2397; found, 475.2387.

4.1.2.15. Compound 15. Yellow solid; yield 59\%; ${ }^{1} \mathrm{H}$ NMR $\left(600 \mathrm{MHz}, \mathrm{CDCl}_{3}\right): \delta 8.07(\mathrm{~d}, J=8.7 \mathrm{~Hz}, 1 \mathrm{H}), 7.98(\mathrm{~d}, J=8.4$ $\mathrm{Hz}, 1 \mathrm{H}), 7.91(\mathrm{~d}, J=8.7 \mathrm{~Hz}, 1 \mathrm{H}), 7.75(\mathrm{~d}, J=7.8 \mathrm{~Hz}, 1 \mathrm{H}), 7.62$ $(\mathrm{t}, J=7.1 \mathrm{~Hz}, 1 \mathrm{H}), 7.46(\mathrm{t}, J=7.0 \mathrm{~Hz}, 1 \mathrm{H}), 7.23(\mathrm{~d}, J=7.4 \mathrm{~Hz}$, $4 \mathrm{H}), 7.21(\mathrm{~s}, 1 \mathrm{H}), 7.11(\mathrm{~s}, 1 \mathrm{H}), 6.99(\mathrm{~s}, 1 \mathrm{H}), 4.81(\mathrm{~d}, J=16.9$ $\mathrm{Hz}, 1 \mathrm{H}), 4.00(\mathrm{~s}, 3 \mathrm{H}), 3.86(\mathrm{~s}, 3 \mathrm{H}), 3.40(\mathrm{~s}, 2 \mathrm{H}), 3.17$ (d, J = $16.9 \mathrm{~Hz}, 1 \mathrm{H}), 2.73(\mathrm{dd}, J=50.0,9.8 \mathrm{~Hz}, 2 \mathrm{H}), 2.43(\mathrm{dd}, J=14.3$, $4.5 \mathrm{~Hz}, 1 \mathrm{H}), 1.98(\mathrm{dd}, J=14.3,6.3 \mathrm{~Hz}, 1 \mathrm{H}), 1.77-1.71(\mathrm{~m}, 4 \mathrm{H})$, $1.63(\mathrm{~d}, J=11.6 \mathrm{~Hz}, 2 \mathrm{H}), 0.85(\mathrm{~d}, J=16.3 \mathrm{~Hz}, 1 \mathrm{H}) .{ }^{13} \mathrm{C} \mathrm{NMR}$ $\left(150 \mathrm{MHz}_{\mathrm{CDCl}}\right.$ ): $\delta 206.2,160.1,156.1,149.8,149.7,147.4$, $136.2,129.7,129.6,129.2,128.3,128.0,127.5,127.3,127.2$, 126.3, 120.5, 107.6, 105.1, 63.4, 61.3, 56.5, 56.3, 53.8, 53.7, 45.3, 37.0, 33.5, 33.1, 31.6. HR-ESI-MS: calcd for $\mathrm{C}_{31} \mathrm{H}_{36} \mathrm{NO}_{4}[\mathrm{M}+$ $\mathrm{H}]^{+}, 486.2644$; found, 486.2629 .

4.2. Biological Materials and Methods. All the biological materials and methods are carried out according to our previous work. $^{24}$

\section{ASSOCIATED CONTENT}

\section{Supporting Information}

The Supporting Information is available free of charge at https://pubs.acs.org/doi/10.1021/acsomega.1c03103.

${ }^{1} \mathrm{H}$ and ${ }^{13} \mathrm{C}$ NMR spectra for all the new compounds (PDF) 


\section{AUTHOR INFORMATION}

\section{Corresponding Authors}

Xian-Li Zhou - School of Life Science and Engineering, Southwest Jiaotong University, Chengdu 610031, PR China; (ㄱ) orcid.org/0000-0002-1690-0578; Email: zhouxl@ swjtu.edu.cn

Feng Gao - School of Life Science and Engineering, Southwest Jiaotong University, Chengdu 610031, PR China; ○ orcid.org/0000-0001-9436-681X; Email: gaof@ swjtu.edu.cn

\section{Authors}

Lin-Xi Wan - School of Life Science and Engineering, Southwest Jiaotong University, Chengdu 610031, PR China; Key Laboratory of Advanced Technology of Materials, Ministry of Education, Southwest Jiaotong University, Chengdu 610031, PR China

Shi-Xing Miao - School of Life Science and Engineering, Southwest Jiaotong University, Chengdu 610031, PR China

Zhen-Xiang $\mathrm{He}-$ School of Life Science and Engineering, Southwest Jiaotong University, Chengdu 610031, PR China

Xiaohuan Li - School of Life Science and Engineering, Southwest Jiaotong University, Chengdu 610031, PR China

Complete contact information is available at:

https://pubs.acs.org/10.1021/acsomega.1c03103

\section{Notes}

The authors declare no competing financial interest.

\section{ACKNOWLEDGMENTS}

This work was finically supported by the Research Foundation for Administration of traditional Chinese Medicine of Sichuan Province (2020HJZX002), NSFC of China (81773605, 31870329, and 82003634), and the Fundamental Research Funds for the Central Universities of China (YGJH2020-LY06).

\section{REFERENCES}

(1) Riverol, M.; Slachevsky, A.; López, O. L. Efficacy and Tolerability of a Combination Treatment of Memantine and Donepezil for Alzheimer's Disease: A Literature Review Evidence. Eur. Neurol. J. 2011, 3, 15-19.

(2) Sahin, Z.; Biltekin, S. N.; Bülbül, E. F.; Yurttas, L.; Berk, B.; Demirayak, Ş. Design and synthesis of new donepezil analogs derived from arylpiperazine scaffold as acetylcholinesterase inhibitors. Phosphorus, Sulfur Silicon Relat. Elem. 2020, 196, 283-293.

(3) Seltzer, B. Donepezil: a review. Expert Opin. Drug Metab. Toxicol. 2005, 1, 527-536.

(4) Seltzer, B. Donepezil: an update. Expert Opin. Pharmacother. 2007, $8,1011-1023$.

(5) Sağlık, B. N.; Levent, S.; Osmaniye, D.; Cevik, U. A.; Cavusoglu, B. K.; Ozkay, Y.; Koparal, A. S.; Kaplancikli, Z. A. Design, Synthesis, and Biological Activity Evaluation of New Donepezil-Like Compounds Bearing Thiazole Ring for the Treatment of Alzheimer's Disease. Crystals 2020, 10, 637.

(6) Asiri, Y. A.; Mostafa, G. A. E. Donepezil. Profiles Drug Subst., Excipients, Relat. Methodol. 2010, 35, 117-150.

(7) Silva, M. A.; Kiametis, A. S.; Treptow, W. Donepezil Inhibits Acetylcholinesterase via Multiple Binding Modes at Room Temperature. J. Chem. Inf. Model. 2020, 60, 3463-3471.

(8) Dias, K. S. T.; de Paula, C. T.; dos Santos, T.; Souza, I. N. O.; Boni, M. S.; Guimarães, M. J. R.; da Silva, F. M. R.; Castro, N. G.; Neves, G. A.; Veloso, C. C.; et al. Design, synthesis and evaluation of novel feruloyl-donepezil hybrids as potential multitarget drugs for the treatment of Alzheimer's disease. Eur. J. Med. Chem. 2017, 130, 440457.
(9) Dias Viegas, F. P.; de Freitas Silva, M.; Divino da Rocha, M.; Castelli, M. R.; Riquiel, M. M.; Machado, R. P.; Vaz, S. M.; Simões de Lima, L. M.; Mancini, K. C.; Marques de Oliveira, P. C.; et al. Design, synthesis and pharmacological evaluation of $\mathrm{N}$-benzyl-piperidinyl-arylacylhydrazone derivatives as donepezil hybrids: Discovery of novel multi-target anti-alzheimer prototype drug candidates. Eur. J. Med. Chem. 2018, 147, 48-65.

(10) Gabr, M. T.; Abdel-Raziq, M. S. Structure-based design, synthesis, and evaluation of structurally rigid donepezil analogues as dual AChE and BACE-1 inhibitors. Bioorg. Med. Chem. Lett. 2018, 28, 2910-2913.

(11) Terra, B. S.; da Silva, P. H. C.; Tramarin, A.; Franco, L. L.; da Cunha, E. F. F.; Macedo Junior, F.; Ramalho, T. C.; Bartolini, M.; Bolognesi, M. L.; de Fatima, A. Two Novel Donepezil-Lipoic Acid Hybrids: Synthesis, Anticholinesterase and Antioxidant Activities and Theoretical Studies. J. Braz. Chem. Soc. 2018, 29, 738-747.

(12) Ismail, M. M.; Kamel, M. M.; Mohamed, L. W.; Faggal, S. I. Synthesis of New Indole Derivatives Structurally Related to Donepezil and Their Biological Evaluation as Acetylcholinesterase Inhibitors. Molecules 2012, 17, 4811-4823.

(13) Alonso, D.; Dorronsoro, I.; Rubio, L.; Muñoz, P.; GarcíaPalomero, E.; Del Monte, M.; Bidon-Chanal, A.; Orozco, M.; Luque, F. J.; Castro, A.; et al. Donepezil-tacrine hybrid related derivatives as new dual binding site inhibitors of AChE. Bioorg. Med. Chem. 2005, 13, $6588-6597$.

(14) Hu, Y.; Zhang, J.; Chandrashankra, O.; Ip, F. C. F.; Ip, N. Y. Design, synthesis and evaluation of novel heterodimers of donepezil and huperzine fragments as acetylcholinesterase inhibitors. Bioorg. Med. Chem. 2013, 21, 676-683.

(15) Huang, C.-S.; Tu, W.-T.; Luo, M.; Shi, J.-C. Molecular Docking and Design of Novel Heterodimers of Donepezil and Huperzine Fragments as Acetylcholinesterase Inhibitors. Chin. J. Struct. Chem. 2016, 35, 839-848.

(16) Wu, M.-Y.; Esteban, G.; Brogi, S.; Shionoya, M.; Wang, L.; Campiani, G.; Unzeta, M.; Inokuchi, T.; Butini, S.; Marco-Contelles, J. Donepezil-like multifunctional agents: Design, synthesis, molecular modeling and biological evaluation. Eur. J. Med. Chem. 2016, 121, 864879.

(17) Mishra, C. B.; Kumari, S.; Manral, A.; Prakash, A.; Saini, V.; Lynn, A. M.; Tiwari, M. Design, synthesis, in-silico and biological evaluation of novel donepezil derivatives as multi-target-directed ligands for the treatment of Alzheimer's disease. Eur. J. Med. Chem. 2017, 125, 736750.

(18) Wang, Z.-M.; Cai, P.; Liu, Q.-H.; Xu, D.-Q.; Yang, X.-L.; Wu, J.-J.; Kong, L.-Y.; Wang, X.-B. Rational modification of donepezil as multifunctional acetylcholinesterase inhibitors for the treatment of Alzheimer's disease. Eur. J. Med. Chem. 2016, 123, 282-297.

(19) Andreani, A.; Cavalli, A.; Granaiola, M.; Guardigli, M.; Leoni, A.; Locatelli, A.; Morigi, R.; Rambaldi, M.; Recanatini, M.; Roda, A. Synthesis and screening for antiacetylcholinesterase activity of (1benzyl-4-oxopiperidin-3-ylidene)methylindoles and -pyrroles related to donepezil. J. Med. Chem. 2001, 44, 4011-4014.

(20) Chaves, S.; Resta, S.; Rinaldo, F.; Costa, M.; Josselin, R.; Gwizdala, K.; Piemontese, L.; Capriati, V.; Pereira-Santos, A. R.; Cardoso, S. M.; et al. Design, Synthesis, and In Vitro Evaluation of Hydroxybenzimidazole-Donepezil Analogues as Multitarget-Directed Ligands for the Treatment of Alzheimer's Disease. Molecules 2020, 25, 985.

(21) Omran, Z.; Cailly, T.; Lescot, E.; Santos, J. S.-d. O.; Agondanou, J.-H.; Lisowski, V.; Fabis, F.; Godard, A.-M.; Stiebing, S.; Le Flem, G.; et al. Synthesis and biological evaluation as AChE inhibitors of new indanones and thiaindanones related to donepezil. Eur. J. Med. Chem. 2005, 40, 1222-1245.

(22) Xu, Y.; Su, T.; Huang, Z.; Dong, G. Practical Direct $\alpha$-Arylation of Cyclopentanones by Palladium/Enamine Cooperative Catalysis. Angew. Chem., Int. Ed. 2016, 55, 2559-2563.

(23) Li, P.; Lü, B.; Fu, C.; Ma, S. Zheda-Phos for General $\alpha$ Monoarylation of Acetone with Aryl Chlorides. Adv. Synth. Catal. 2013, $355,1255-1259$. 
(24) Wan, L.-X.; Zhen, Y.-Q.; He, Z.-X.; Zhang, Y.; Zhang, L.; Li, X.; Gao, F.; Zhou, X.-L. Late-Stage Modification of Medicine: PdCatalyzed Direct Synthesis and Biological Evaluation of N-Aryltacrine

Derivatives. ACS Omega 2021, 6, 9960-9972. 